\title{
Chemical evolution of the Galactic bulge: different stellar populations and possible gradients
}

\author{
V. Grieco ${ }^{1}$, F. Matteucci ${ }^{1,2}$, A. Pipino ${ }^{3}$, and G. Cescutti ${ }^{4}$ \\ 1 Dipartimento di Fisica, Sezione di Astronomia, Università di Trieste, via G.B. Tiepolo 11, 34131 Trieste, Italy \\ e-mail: grieco@oats.inaf.it \\ 2 INAF Osservatorio Astronomico di Trieste, via G.B. Tiepolo 11, 34131 Trieste, Italy \\ ${ }^{3}$ Institut fur Astronomie, ETH Zurich, Wolfgang-Pauli-Str. 27, 8093 Zurich, Switzerland \\ ${ }^{4}$ Leibniz-Institut für Astrophysik Potsdam (AIP), An der Sternwarte 16, 14482 Potsdam, Germany
}

Received 6 June 2012 / Accepted 14 September 2012

\begin{abstract}
Context. The recent although controversial discovery of two main stellar populations in the Galactic bulge, one metal-poor with a spheroid kinematics and the other metal-rich with a bar-like kinematics, suggests a revision of the classical model for bulge formation. Aims. We aim at computing in detail the chemical evolution of the Galactic bulge in order to explain the existence of the two main stellar populations. We also plan to explore the possible existence of spatial abundance gradients inside the bulge.

Methods. To do that, we adopt a chemical evolution model that follows the evolution of several chemical species (from $\mathrm{H}$ to Ba) and takes into account both infall and outflow of gas. We assume that the metal-poor population formed first and on a short timescale, in agreement with previous models, while the metal-rich population formed later and out of the enriched gas either left from the formation of the previous one or originating from the inner disk. We predict the stellar distribution functions for Fe and $\mathrm{Mg}$, the mean $\langle[\mathrm{Fe} / \mathrm{H}]\rangle$ and $\langle[\mathrm{Mg} / \mathrm{H}]\rangle$ as well as the $[\mathrm{Mg} / \mathrm{Fe}]$ vs. $[\mathrm{Fe} / \mathrm{H}]$ relations in the two stellar populations. Then, we consider the case in which the metal-poor population could be the result of sub-populations formed with different chemical enrichment rates. In particular, the population close to the Galactic center could have evolved very fast, while the more external population could have evolved more slowly, in agreement with the dissipational gravitational collapse scenario.

Results. When compared with observations, our results confirm that the old more metal-poor stellar population formed very fast (on a timescale of $0.1-0.3 \mathrm{Gyr}$ ) by means of an intense burst of star formation coupled with an initial mass function flatter than in the solar vicinity, but not as flat as suggested in previous works. The metal-rich population, instead, should have formed on a longer timescale ( $\sim 3 \mathrm{Gyr}$ ). We predict differences in the mean abundances of the two populations: in particular, we find a difference of $\sim-0.52$ dex for $\langle[\mathrm{Fe} / \mathrm{H}]\rangle$. These differences can be interpreted as a metallicity gradient. We also predict possible gradients for $\mathrm{Fe}, \mathrm{O}, \mathrm{Mg}, \mathrm{Si}, \mathrm{S}$, and $\mathrm{Ba}$ between sub-populations inside the metal-poor population itself (e.g., -0.145 dex for $\langle[\mathrm{Fe} / \mathrm{H}]\rangle$ ). Finally, by means of a chemodynamical model following a dissipational collapse, we predict a gradient inside $500 \mathrm{pc}$ from the Galactic center of $-0.26 \mathrm{dex} \mathrm{kpc}^{-1}$ in Fe.

Conclusions. We conclude that the chemical evolution of the Galactic bulge, as suggested by its stellar populations, has been quite complex. A stellar population forming by means of a classical gravitational gas collapse is probably mixed with a younger stellar population created perhaps by the bar evolution. The differences among their mean abundances can be interpreted as a gradient. On the basis of both chemical and chemo-dynamical models, we also conclude that it is possible that the metal-poor population itself contains abundance gradients and thus different stellar populations.
\end{abstract}

Key words. Galaxy: abundances - Galaxy: bulge - Galaxy: evolution

\section{Introduction}

Recent data concerning the Galactic bulge are suggesting a rather complex picture for its formation. Recently, Babusiaux et al. (2010), Gonzalez et al. (2011), Bensby et al. (2011), Hill et al. (2011), Robin et al. (2012) and Uttenthaler et al. (2012) have shown that the stellar populations and chemical evolution of the Galactic bulge are not as simple as it has appeared up to now. In particular, Bensby et al. (2011) observed microlensed dwarfs and subgiant bulge stars and concluded that their distribution is bimodal with one peak at $[\mathrm{Fe} / \mathrm{H}]=-0.6$ dex and one peak at $[\mathrm{Fe} / \mathrm{H}]=0.3$ dex. Hill et al. (2011) by studying bulge red clump stars also found two distinct stellar populations in the bulge, one with a peak at $[\mathrm{Fe} / \mathrm{H}] \sim-0.45 \mathrm{dex}$ and another with a peak at $[\mathrm{Fe} / \mathrm{H}] \sim+0.3$ dex. The interpretation of these two populations is that the metal-poor (MP) one probably reflects the classical bulge component, the old spheroid population formed on a short timescale, as witnessed by the high $[\mathrm{Mg} / \mathrm{Fe}] \sim+0.3$ dex ratio and the kinematics corresponding to an old spheroid (Babusiaux et al. 2010), whereas the metalrich (MR) population seems to possess a bar kinematics and could have originated by a pre-enriched gas coming either from the residual gas from the formation of the MP component or from the metal rich inner disk. These stars could have formed on a longer timescale than the MP component, as witnessed by their almost solar $[\mathrm{Mg} / \mathrm{Fe}]$ ratios. As is well known, in a regime of a very fast star formation rate, most of the stars form with high $[\mathrm{Mg} / \mathrm{Fe}]$ ratios, due to the predominant pollution by corecollapse supernovae $(\mathrm{SNe})$. On the other hand, in a regime of slow star formation, the $[\mathrm{Mg} / \mathrm{Fe}]$ ratios tend to be lower, due to 
the pollution by Type Ia SNe intervening later than core-collapse $\mathrm{SNe}$ in the chemical enrichment process.

What emerges from the recent data is therefore that the Galactic bulge could have both the characteristics of a classical bulge and a pseudo-bulge. On the one hand, the existence of a bar is now proven by several studies (e.g., McWilliam \& Zoccali 2010; Nataf et al. 2010; Saito et al. 2011), suggesting that the bulge has an $\mathrm{X}$-shaped structure, which can indicate the existence of a bar. The extensive survey by Ness \& Freeman (2012) has shown that the Milky Way bulge is indeed a bar. Results from BRAVA survey (e.g., Rich et al. 2007a) did not find evidence for a different population from the bar one, whereas Shen et al. (2010) and Kunder et al. (2011) suggested that the classical bulge component exists, but is $<8 \%$ of the mass of the disk. On the other hand, color-magnitude diagram analyses of bulge stars (e.g., Zoccali et al. 2003; Kuijken \& Rich 2002; Clarkson et al. 2008, 2011) have indicated that the bulge is old and that there is little age spread among stars. This fact, coupled with high $[\mathrm{Mg} / \mathrm{Fe}]$ ratios, argues in favor of fast bulge formation. Therefore, the situation seems to be quite complex and even contradictory.

Various theories for bulge formation have been put forward in the past few years. Wyse \& Gilmore (1992) first summarized the various possibilities, and in the following years many studies have appeared on the subject. The main proposed scenarios are as follows:

- a) Accretion of stellar satellites. This idea was later developed in models where the bulge formed by accretion of extant stellar systems, which hierarchically merged and eventually settled in the center of the Galaxy (Noguchi 1999; Aguerri et al. 2001; Bournaud et al. 1999).

- b) In situ star formation from primordial or slightly enriched gas. The bulge was formed by a fast gravitational collapse (Larson 1976) or slow accumulation of gas at the center of the Galaxy and subsequent evolution with either fast or slow star formation; the accreting gas could have been primordial or metal enriched by the halo, thick-disk, or thindisk. In the past few years, the comparison between model predictions and the observed metallicity distribution function (MDF) of the Galactic bulge suggested that this component of the Milky Way had evolved very fast and with a flatter initial mass function (IMF) than in the solar vicinity (e.g., Matteucci \& Brocato 1990; Matteucci et al. 1999; Ferreras et al. 2003; Ballero et al. 2007; Cescutti \& Matteucci 2011).

- c) Secular evolution. The bulge formed as a result of secular evolution of the disk through a bar forming a pseudo-bulge (e.g., Combes et al. 1990; Norman et al. 1996; Kormendy $\&$ Kennicutt 2004; Athanassoula 2005). After the formation of the bar, the bulge heats in the vertical direction giving rise to the typical boxy/peanut configuration. A more recent model belonging to this category assumes that the bulge is formed through bar instability from a disk composed of thin- and thick-disk components (Bekki \& Tsujimoto 2011). However, these models were not tested on the observed chemical abundances.

- d) Mixed scenario: secular and spheroidal components together. Samland \& Gerhard (2003) had predicted, by means of a dynamical model, the existence of two bulge populations, one formed in an early collapse and the other formed late in the bar. Although a two-step formation of the bulge is not a new idea (see Wyse \& Gilmore 1992), Tsujimoto \& Bekki (2012) recently tried to model the two main stellar populations found in the bulge and suggested that the
MP component formed on a timescale of $1 \mathrm{Gyr}$ with a flat IMF $(x=1.05)$, whereas the other component, the MR one, formed from pre-enriched gas on a timescale of 4 Gyr.

In this paper, we aim at computing the chemical evolution of the Galactic bulge by means of a very detailed chemical evolution model following the evolution of 36 chemical species to see whether we are able to reproduce the two observed main stellar components (Hill et al. 2011) and their abundances under reasonable assumptions. In particular, we aim at testing whether the MP population can be explained by a less flat IMF than suggested by Ballero et al. (2007) and Cescutti \& Matteucci (2011) on the basis of the previous observed stellar MDFs available up to now. Then, we will study the differences among the mean abundances of several chemical elements in the two populations. In principle, these differences can be interpreted as abundance gradients, although the two populations are likely to be spatially mixed.

Moreover, we intend to compute possible abundance gradients inside the MP population and see whether they are compatible with the data. The observational situation is, in fact, not yet clear. Rich et al. (2012) did not find any vertical gradient from the Galactic center to the Baade's window inside the innermost $600 \mathrm{pc}$. If true, this suggests that the Baade's window stellar population formed indeed very fast, so that no gradient could be created. In fact, an abundance gradient is naturally created during a dissipative collapse (Larson 1976). On the other hand, Zoccali et al. $(2008,2009)$ found different mean Fe abundances by analyzing different fields in the bulge: in the Baade's window field, they found $\langle[\mathrm{Fe} / \mathrm{H}]\rangle=+0.03$ dex at $b=-4^{\circ}$, while in a field at higher latitude $\left(b=-12^{\circ}\right)$, they found $\langle[\mathrm{Fe} / \mathrm{H}]\rangle=-0.40$ dex. This difference can clearly be interpreted as a vertical gradient along the bulge minor axis.

Pipino et al. (2008) run $1 \mathrm{D}$ chemo-dynamical models for a Milky Way-like bulge $\left(M_{*} \sim 2 \times 10^{10} M_{\odot}\right.$ and $\left.R_{\text {eff }}=1 \mathrm{kpc}\right)$ and found that during the gravitational collapse giving rise to the bulge, an abundance gradient in the stars is indeed created. In particular, they suggested that inside $1 \mathrm{kpc}$ from the Galactic center, we should expect a gradient in the global stellar metallicity of $\mathrm{d}[\mathrm{Z} / \mathrm{H}] / \mathrm{d} R=-0.22 \mathrm{dex}_{\mathrm{kpc}}-1$. Here, we will rerun this model and give predictions for the gradients of $\mathrm{O}$ and $\mathrm{Fe}$. Moreover, in a simple way we will explore if inside the MP bulge component we can identify at least two sub-populations, formed at different rates and showing different average abundances.

Finally, it is worth noting that we will concentrate on explaining the observed metallicity distribution and chemical abundances of Galactic bulge stars and that we cannot say much about secular evolution and bar formation, since our model does not take into account stellar dynamics. Galactic chemical evolution can only put constraints on the timescales of formation of the different stellar populations, but it cannot predict how the bulge actually formed. For this reason, in this paper we are concerned only with chemical abundances.

The paper is organized as follows: in Sect. 2, we describe the observational data, while in Sect. 3 we present the chemical evolution model. In Sect. 4, the results are depicted and compared with observations. Finally, in Sect. 5, we present a discussion on bulge formation and draw some conclusions.

\section{The observational data}

In this paper, we compare our results with the recent data from Hill et al. (2011). In that paper, they presented measures of 
$[\mathrm{Fe} / \mathrm{H}]$ for 219 bulge red clump stars from $R=20000$ resolution spectra obtained with FLAMES/GIRAFFE at the Very Large Telescope (VLT). For a subsample of 162 stars, they also measured $[\mathrm{Mg} / \mathrm{H}]$. The stars are all in a Baade's window. They interpreted the iron distribution in bulge stars as bimodal, indicating two different stellar components of equal size: a metalpoor component centered around $[\mathrm{Fe} / \mathrm{H}]=-0.30 \mathrm{dex}$ and $[\mathrm{Mg} / \mathrm{H}]=-0.06$ dex with a large dispersion and a metal-rich narrow component centered around $[\mathrm{Fe} / \mathrm{H}]=+0.32$ dex and $[\mathrm{Mg} / \mathrm{H}]=+0.35$ dex. Therefore, the MP component shows high average $[\mathrm{Mg} / \mathrm{Fe}] \sim 0.3 \mathrm{dex}$, whereas the MR one shows $[\mathrm{Mg} / \mathrm{Fe}] \sim 0$ dex. Hill et al. (2011) discussed the possible contamination of the two populations by stars of the inner disk and halo and concluded that it is very small, although the situation is still uncertain (see also Bensby et al. 2011). In a previous paper, Babusiaux et al. (2010) also found kinematical differences among these two components: the MP component shows a kinematics typical of an old spheroid (classical bulge), whereas that of the MR component is consistent with a bar population (pseudo-bulge). Bensby et al. (2011) measured detailed abundances of 26 microlensed dwarf and subgiant stars in the Galactic bulge; in particular, the stars are all located between Galactic latitudes $-2^{\circ}$ to $-5^{\circ}$, similar to Baade's window at $(l, b)=\left(1^{\circ},-4^{\circ}\right)$. The analysis was based on highresolution spectra obtained with UVES/VLT. They also showed that the bulge MDF is double-peaked; one peak at $[\mathrm{Fe} / \mathrm{H}]=$ $-0.6 \pm 0.3$ dex, lower than the peak of the MP population of Hill et al. (2011), and one at $[\mathrm{Fe} / \mathrm{H}]=+0.32 \pm 0.16$ dex. Clearly, the most recent observational evidence points toward the existence of two main populations in the bulge, although the sample of dwarf stars needs to be substantially enlarged before drawing firm conclusions.

\section{The chemical evolution model}

Here we will try to model the two stellar populations (MP and MR), as described in the previous section. The chemical evolution model is similar to that adopted by Cescutti \& Matteucci (2011), which is an upgraded version of that of Ballero et al. (2007), where a detailed description can be found. We remind here that the model can follow in detail the evolution of several chemical elements, including H, D, He, Li, C, N, O, $\alpha$-elements, $\mathrm{Fe}$ and Fe-peak elements, s- and r-process elements. The IMF is assumed to be constant in space and time, and it is allowed to vary in order to test which one best fits the MDF. The star formation rate adopted for the bulge is a simple Schmidt law with exponent $k=1$. The star formation rate per unit mass of gas (efficiency of star formation), is allowed to vary from $v=2$ to $25 \mathrm{Gyr}^{-1}$. In particular, to model the MP old spheroid component, we adopt $v=25 \mathrm{Gyr}^{-1}$, whereas for the MR pseudo-bulge component, $v=2 \mathrm{Gyr}^{-1}$. This model takes stellar feedback into account and computes the thermal energy injected into the interstellar medium (ISM) by SNe, as described in Ballero et al. (2007).

We assume that both stellar populations formed during episodes of gas accretion: the law for gas accretion is assumed to be the same in both cases, but the abundances of the infalling gas are different. We suppose that the gas that formed the MP component was primordial or slightly enriched from the halo formation, whereas the gas that formed the MR component was substantially enriched. In particular, the assumed chemical composition of the gas out of which the MR component formed has a $[\mathrm{Fe} / \mathrm{H}]=-0.6 \mathrm{dex}$, and all the abundance ratios reflect the composition of the gas forming the MP component at $t \sim 0.06 \mathrm{Gyr}$.
It is worth noting that this particular chemical composition is similar to the composition of the gas in the innermost regions of the Galactic disk, near $2 \mathrm{kpc}$, at an age of $\sim 2$ Gyr. The reason for the greater age is that the inner disk must have evolved more slowly than the classical bulge, with a lower star formation rate. Therefore, the MR population could have started to form with a delay of 2 Gyr relative to the MP population and from gas of the inner disk.

The assumed gas accretion law is

$\dot{G}_{i}(t)_{\text {inf }}=A(r) X_{\text {inf }} \sigma\left(t_{G}\right) \mathrm{e}^{-t / \tau}$

where $i$ represents a generic chemical element, $\tau$ is an appropriate collapse timescale fixed by reproducing the observed stellar MDF, and $A(r)$ is a parameter constrained by the requirement of reproducing the current total surface mass density in the Galactic bulge $\left(\sigma_{\text {bulge }} \sim 1300 M_{\odot} \mathrm{yr}^{-1}\right)$, which in turn gives a total bulge mass of $\sim 2.0 \times 10^{10} M_{\odot}$ for a bulge radius of $R_{\mathrm{B}}=2 \mathrm{kpc}$ and surface mass density distribution following a Sersic profile (see Ballero et al. 2007). Finally, $X_{\text {inf }}$ are the abundances of the infalling gas, considered constant in time, and $t_{G}$ is the Galactic lifetime (13.7 Gyr). In particular, the abundances of the infalling gas are considered either primordial or slightly enriched at the level of the average metallicity of the halo stars $(\langle[\mathrm{Fe} / \mathrm{H}]\rangle=-1.5 \mathrm{dex})$. This second option is justified if we think that the bulge stars formed out of gas lost from the halo and/or the inner thick-thin disk.

The IMFs adopted here are the one suggested by Ballero et al. (2007)

$\phi(m) \propto m^{-(1+x)}$

with $x=0.95$ for $M>1 M_{\odot}$ and $x=0.33$ for $M<1 M_{\odot}$ in the mass range $0.1-100 M_{\odot}$; the normal Salpeter IMF $(x=1.35)$ in the mass range $0.1-100 M_{\odot}$, and the two-slope Scalo (1986) IMF, as adopted in Chiappini et al. (1997, 2001), with $x=1.35$ for $m<2 M_{\odot}$ and $x=1.7$ for $m \geq 2 M_{\odot}$, always in the mass range $0.1-100 M_{\odot}$.

\subsubsection{Nucleosynthesis and stellar evolution prescriptions}

For the evolution of ${ }^{7} \mathrm{Li}$, we followed the prescriptions of Romano et al. (1999), who predicted the evolution of Li abundance in the Galactic bulge. The main Li producers assumed in that model are i) core-collapse $\mathrm{SNe}$; ii) massive-AGB stars; iii) C-stars; iv) novae; and v) cosmic rays. We refer the reader to this paper for details.

For all the other elements, we adopted the same yields as in Cescutti \& Matteucci (2011). In particular, detailed nucleosynthesis prescriptions are taken from François et al. (2004), who made use of widely adopted stellar yields and compared the results obtained by including these yields in a detailed chemical evolution model with observational data with the aim of constraining stellar nucleosynthesis. For low- and intermediatemass $\left(0.8-8 M_{\odot}\right)$ stars, which produce ${ }^{12} \mathrm{C}$ and ${ }^{14} \mathrm{~N}$, yields are taken from the standard model of van den Hoek \& Groenewegen (1997) as a function of the initial stellar metallicity. Concerning massive stars $\left(M>10 M_{\odot}\right)$, in order to best fit the data in the solar neighbourhood when adopting Woosley \& Weaver (1995) yields, François et al. (2004) found that: Mg yields should be increased in stars with masses $11-20 M_{\odot}$ and decreased in stars larger than $20 M_{\odot}$, while Si yields should be slightly increased in stars above $40 M_{\odot}$. In the range of massive stars, we also adopted the yields of Maeder (1992) and Meynet \& Maeder (2002) containing mass loss. The effect of mass loss is visible only for 
Table 1. Model parameters.

\begin{tabular}{lccc}
\hline \hline Model & $v\left(\mathrm{Gyr}^{-1}\right)$ & $\tau(\mathrm{Gyr})$ & IMF \\
\hline MP & 25 & 0.1 & Salpeter \\
MR & 2 & 3.0 & Salpeter \\
IMP & 25 & 0.1 & Salpeter \\
EMP & 10 & 0.1 & Salpeter \\
\hline
\end{tabular}

metallicities $\geq Z_{\odot}$. The use of these yields is particularly important for studying the evolution of $\mathrm{O}$ and $\mathrm{C}$, the two most affected elements (see McWilliam et al. 2008; Cescutti et al. 2009). For $\mathrm{Ba}$, we use the nucleosynthesis prescriptions adopted by Cescutti et al. (2006) to best fit the observational data for this neutron capture element in the solar vicinity; the same nucleosynthesis prescriptions also give good results when applied to dwarf spheroidals (Lanfranchi et al. 2006) and to the Galactic halo using an inhomogenous model (Cescutti 2008). In particular, we assume that the s-process fraction of $\mathrm{Ba}$ is produced in low-mass stars (1-3 $M_{\odot}$, see Busso et al. 2001), whereas the r-process fraction of $\mathrm{Ba}$ originates from stars in the range $12-30 M_{\odot}$.

\section{Results}

\subsection{The two main populations}

We ran several numerical models by varying the most important parameters: the IMF, the efficiency of star formation, and the timescale and chemical composition of the infalling gas. We found that the best models have the following characteristics:

i) MP population is obtained by means of very efficient star formation $\left(v=25 \mathrm{Gyr}^{-1}\right)$ and a very short timescale for infall $(\tau=0.1 \mathrm{Gyr})$, as in our previous papers (e.g., Ballero et al. 2007; Cescutti \& Matteucci 2011), but with a Salpeter IMF, that is less flat than the IMF suggested in Ballero et al. (2007). The reason for this choice is that with a Scalo (1986) IMF, the peak of the MDF of the MP population occurs at $[\mathrm{Fe} / \mathrm{H}] \sim-0.6 \mathrm{dex}$, too low compared with the observed one. On the other hand, the Ballero et al. (2007) IMF predicts a peak at a too high metallicity, $[\mathrm{Fe} / \mathrm{H}] \sim 0$. The model with Salpeter IMF, instead, can well reproduce the MDF of the MP population. The chemical composition of the infalling gas is assumed to be slightly pre-enriched $(\langle[\mathrm{Fe} / \mathrm{H}]\rangle \sim-1.5 \mathrm{dex})$. The infall of primordial gas, in fact, would predict too many metal poor stars in the MDF.

ii) MR population is obtained with a less efficient star formation $\left(v=2 \mathrm{Gyr}^{-1}\right)$ and a longer infall timescale $(\tau=3 \mathrm{Gyr})$, the Salpeter IMF and enriched infall. In particular, for the infalling gas, we assume that the chemical composition corresponds to the gas at an age of $0.06 \mathrm{Gyr}$ since the beginning of the formation of the MP population. This composition also represents the metallicity of the gas in the very inner disk at an age of $2 \mathrm{Gyr}$, as predicted by chemical evolution models of the Milky Way disk (Cescutti et al. 2007). The predicted metallicity distribution of our best model for the metal-poor population (MP in Table 1) is in reasonable agreement with the observed one (see Fig. 1). In Fig. 2 we show the same MDFs of Fig. 1 but convolved with a Gaussian taking into account an average observational error of 0.25 dex, in agreement with Hill et al. (2011). In Fig. 3 we show our predictions for the MDFs of the MP and MR populations as functions of $[\mathrm{Mg} / \mathrm{H}]$, and in this case we also find good agreement with data. In Fig. 4 we show the same

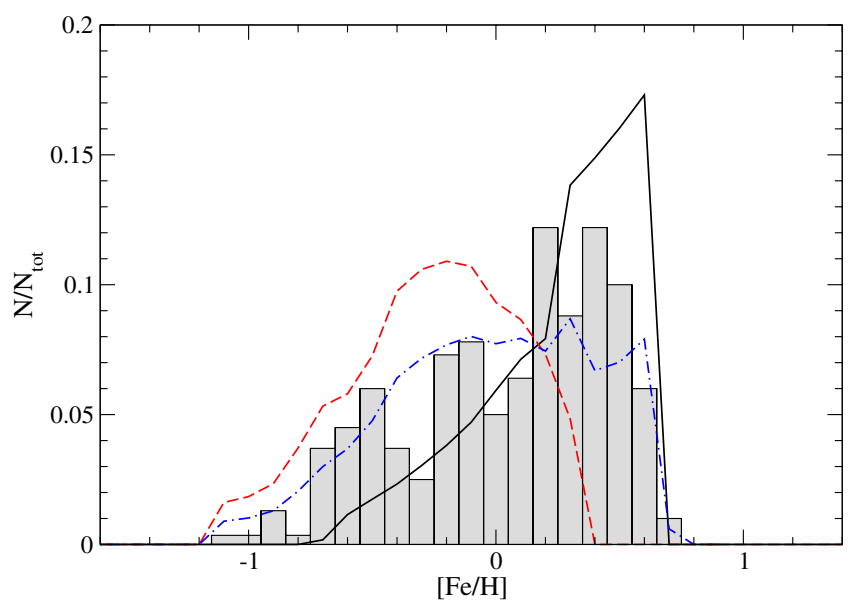

Fig. 1. Predicted MDF for the two populations as functions of $[\mathrm{Fe} / \mathrm{H}]$ : MP (dashed red line) and MR (continuous black line), compared to the data of Hill et al. (2011). The sum of the two distributions is also shown (blue dashed-dotted line).

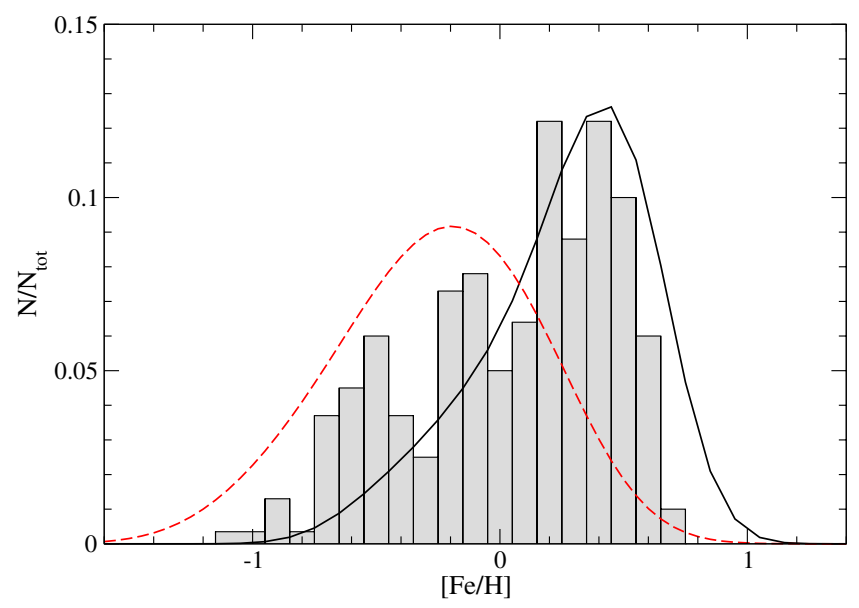

Fig. 2. Predicted and observed MDF for the two populations as in Fig. 1, but the MDFs have been convolved with a Gaussian to take into account an observational error of 0.25 dex.

distributions as in Fig. 3 but convolved with a Gaussian taking into account an observational error of 0.20 dex.

In Fig. 5 we show the predicted $[\mathrm{Mg} / \mathrm{Fe}]$ for the two populations. Clearly, in the MP population, the majority of stars has a high $[\mathrm{Mg} / \mathrm{Fe}]$ roughly constant for a large range of $[\mathrm{Fe} / \mathrm{H}]$; in particular, the $[\mathrm{Mg} / \mathrm{Fe}]$ ratio starts declining at $[\mathrm{Fe} / \mathrm{H}] \geq-0.3$ dex. In the MR population, however, the $[\mathrm{Mg} / \mathrm{Fe}]$ varies from $+0.2 \mathrm{dex}$ to -0.1 dex in a $[\mathrm{Fe} / \mathrm{H}]$ range of $[-0.5-+0.7]$ dex. This lower $[\mathrm{Mg} / \mathrm{Fe}]$ is due to the fact that this population formed out of pre-enriched gas where the pollution from Type Ia $\mathrm{SNe}$ was already present. We note that the predicted $[\mathrm{Mg} / \mathrm{Fe}]$ is slightly high at low metallicities. This is probably due to the assumed $\mathrm{Mg}$ yields in massive stars. The Mg yields are, in fact, still quite uncertain. By lowering the Mg yields, the predicted curve would run lower than it does now, but it would not change its shape. We have computed the mean $\mathrm{Fe}$ abundance, $\langle[\mathrm{Fe} / \mathrm{H}]\rangle$, for the two populations: for the MP one, we find $\langle[\mathrm{Fe} / \mathrm{H}]\rangle=-0.26 \mathrm{dex}$ in very good agreement with Hill et al. (2011), whereas for the MR one, we find $\langle[\mathrm{Fe} / \mathrm{H}]\rangle=+0.26$ dex. This difference in the mean Fe abundance of the two populations could be interpreted as a gradient itself, as suggested by Babusiaux et al. (2010), although it is not clear how the stars of the two populations are spatially distributed and mixed. It is interesting to note that we do not find 
V. Grieco et al.: Chemical evolution of the Galactic bulge: different stellar populations and possible gradients

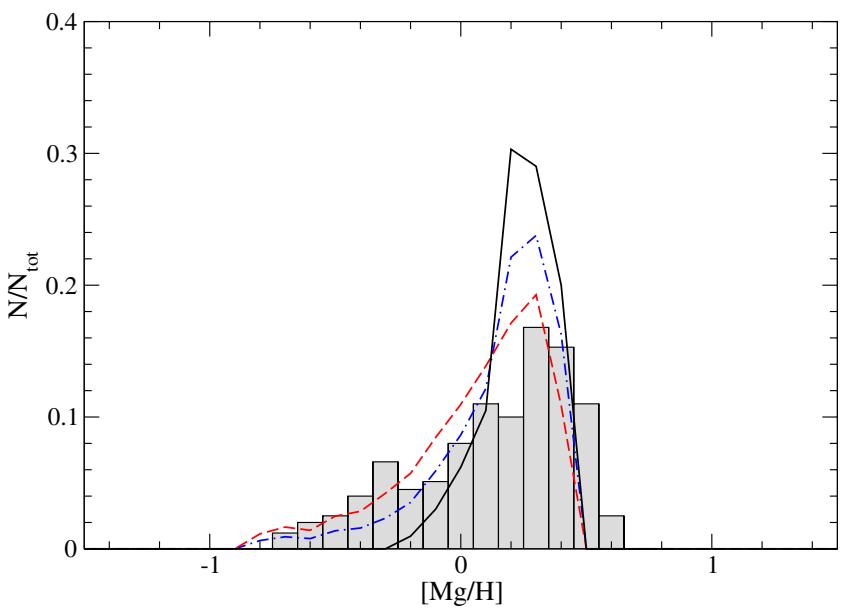

Fig. 3. Predicted MDF for the two populations: MP (red dashed line) and MR (black continuous line) as functions of $[\mathrm{Mg} / \mathrm{H}]$. The data are from Hill et al. (2011). The sum of the two distributions is also shown (blue dashed-dotted line).

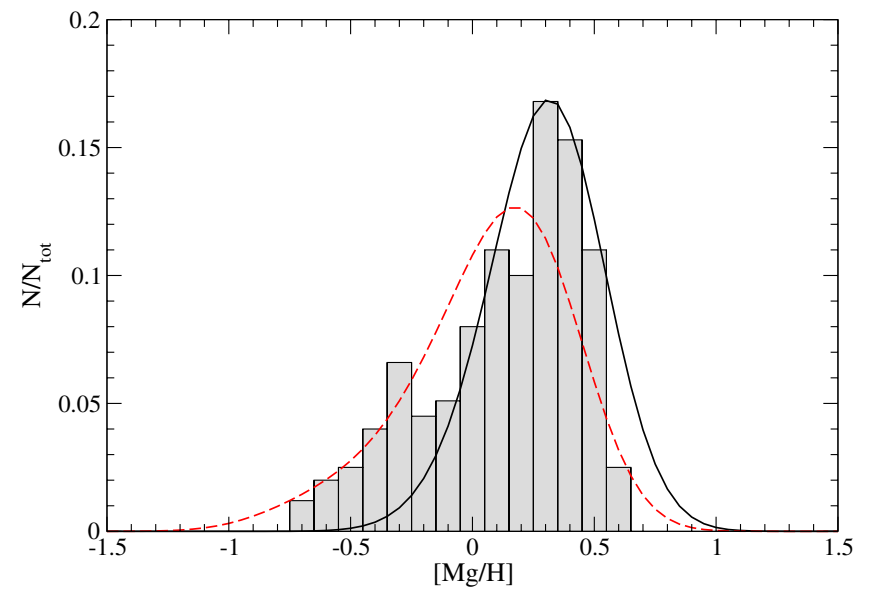

Fig. 4. Predicted MDF for the two populations as a function of $[\mathrm{Mg} / \mathrm{H}]$ as in Fig. 3 but convolved with a Gaussian corresponding to an observational error of $0.2 \mathrm{dex}$.

any development of a galactic wind during the formation of both components. This is due to the deep Galactic potential well in which the bulge is sitting, at variance with what is found for an elliptical galaxy of the same mass as the Galactic bulge, which sits in a shallower potential well (Pipino \& Matteucci 2004).

\subsection{Possible abundance gradient in the metal-poor population?}

The existence of abundance gradients in the Galactic bulge is a very important issue. Minniti et al. (1995) suggested the existence of an abundance gradient in the inner $2 \mathrm{kpc}$, but more recent analyses did not confirm this finding (Ramirez et al. 2000; Rich et al. 2007b; Johnson et al. 2011, 2012; Rich et al. 2012). In particular, these latter studies suggested the absence of a gradient from the Galactic center out to Baade's window. However, Zoccali et al. (2008, 2009) found an abundance gradient along the bulge minor axis, as one moves from Baade's window to $b=-12^{\circ}$. Such a gradient could be due to the formation of the bulge by dissipational collapse, with chemical enrichment being faster in the innermost regions. Here, we test the idea that there could be a gradient inside the MP bulge population. We computed two models describing two different sub-populations

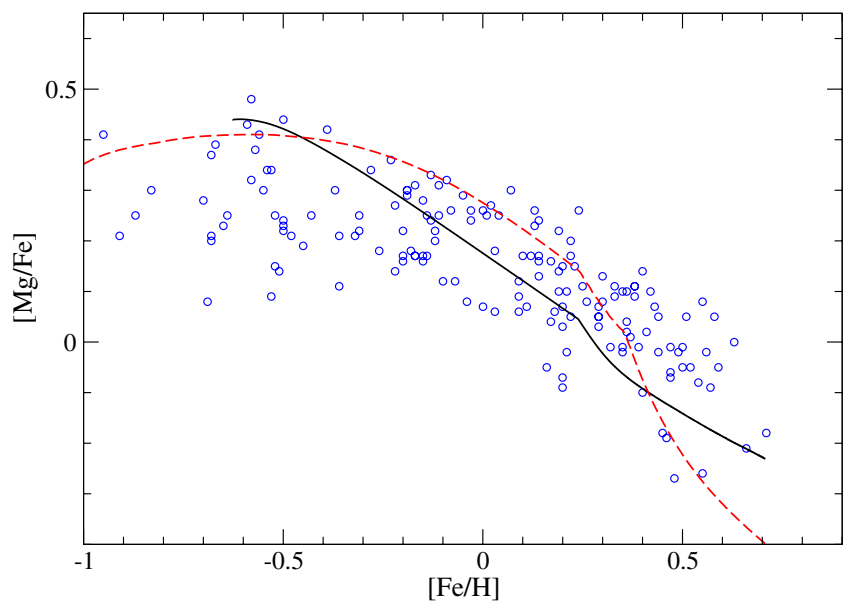

Fig. 5. Predicted $[\mathrm{Mg} / \mathrm{Fe}]$ vs. $[\mathrm{Fe} / \mathrm{H}]$ for the MP and MR populations: MP (dashed red line) and MR (continuous black line), compared with data from Hill et al. (2011) containing both populations.

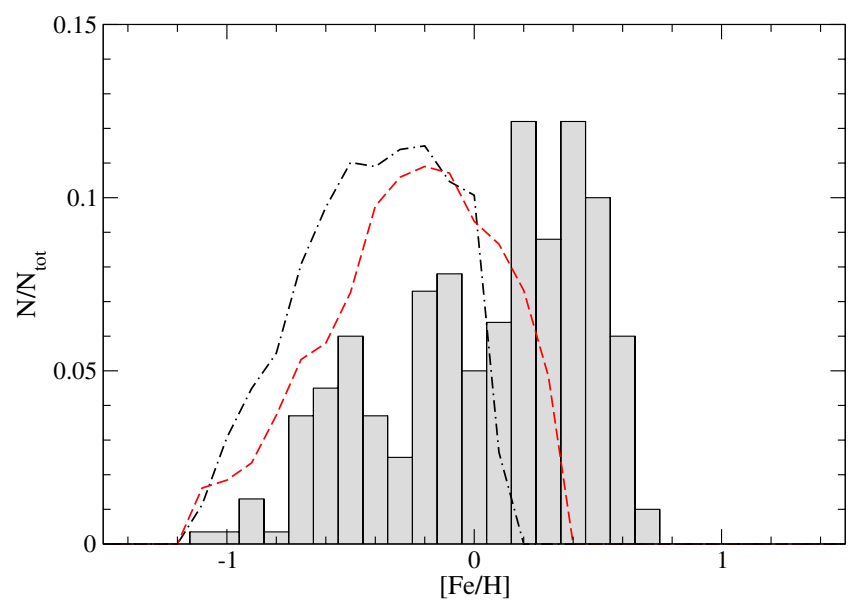

Fig. 6. Predicted MDFs for the two possible sub-populations: IMP (red dashed line) and EMP (black dashed-dotted line) of the MP bulge population. The data are from Hill et al. (2011).

of the MP component: a) the model for the innermost region has the same parameters as those adopted for the MP population ( $v=25 \mathrm{Gyr}^{-1} ; \tau=0.1 \mathrm{Gyr}$; Salpeter IMF), but restricted to the inner $0.6 \mathrm{kpc}$, whereas the model for the outer region (from 0.6 to $\sim 2 \mathrm{kpc})$ has a lower star formation efficiency $\left(v=10 \mathrm{Gyr}^{-1}\right)$, the same timescale for infall, and the same IMF as the inner population. In Table 1, we summarize the model parameters for the four populations: 1) the MP old spheroid population; 2) the MR bar population; 3) the innermost sub-population of the MP called IMP; and 4) the outermost sub-population of the MP called EMP. The predicted MDFs for the two sub-populations (IMP and EMP) are shown in Fig. 6, where they are compared with the observed global MDF. In Fig. 7, we show the same MDFs but convolved with a Gaussian with an average observational error of 0.25 dex, while in Fig. 8 we present the resulting MDF obtained by summing the two MDFs of Fig. 7. The resulting MDF practically coincides with that of the MP population. The two distributions are very similar, although they peak at different $[\mathrm{Fe} / \mathrm{H}]$ values. In particular, the difference between the mean Fe abundance of the IMP and that of the EMP is $\Delta\langle[\mathrm{Fe} / \mathrm{H}]\rangle=-0.145$ dex. Zoccali et al. $(2008,2009)$ suggest $\Delta\langle[\mathrm{Fe} / \mathrm{H}]\rangle=-0.15$ dex going from $b=-4^{\circ}$ up to $b=-12^{\circ}$ (namely, from $600 \mathrm{pc}$ up to $\sim 1.6 \mathrm{kpc}$, in terms of galactocentric distance), in very good agreement with our prediction. Our 


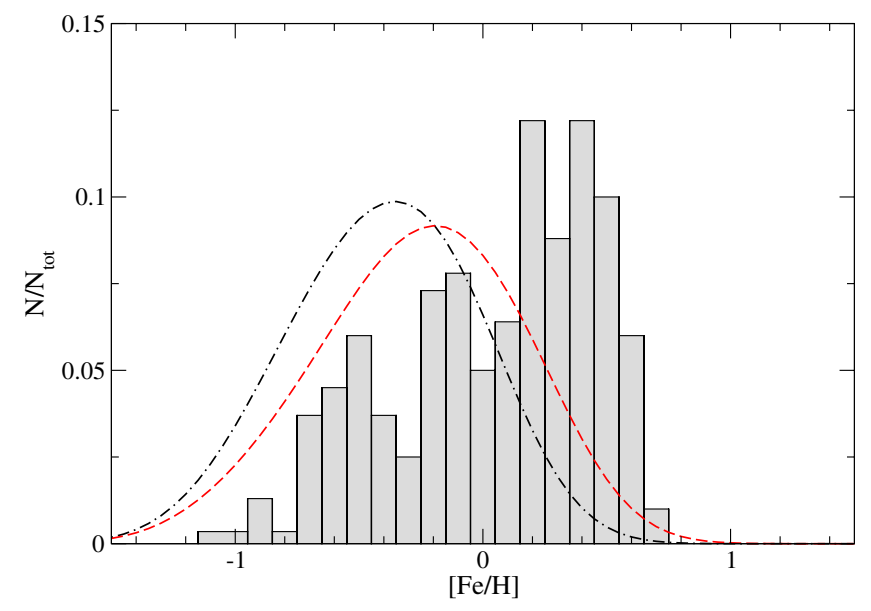

Fig. 7. Predicted MDFs for the two possible sub-populations of the MP bulge population as in Fig. 6, but convolved with a Gaussian with an average observational error of $0.25 \mathrm{dex}$.

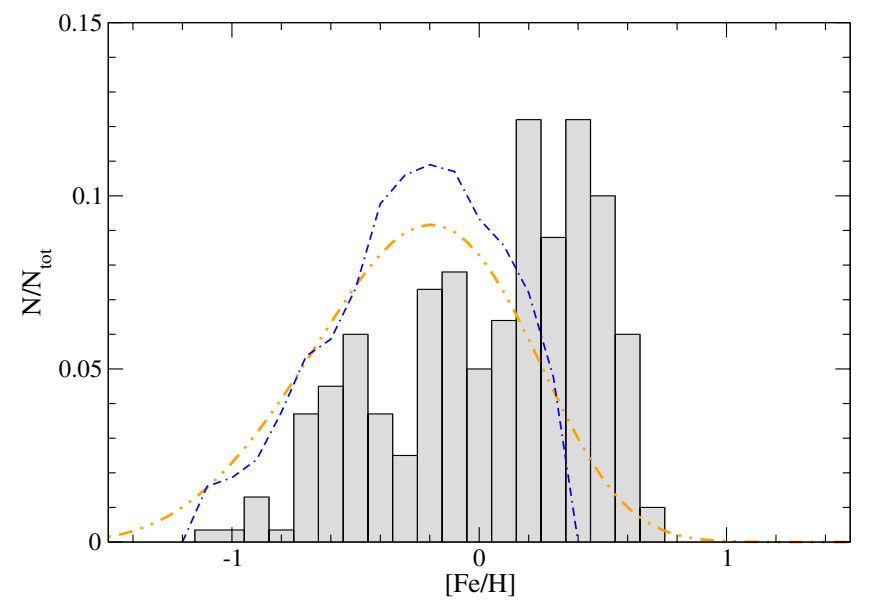

Fig. 8. Predicted global MDF obtained by summing the EMP and IMP populations (dashed-dotted curve). The global MDF is shown also in the Gaussian convolved form (dashed-two-dotted curve).

numerical models show that sub-populations with a larger gradient are not compatible with the observed MDF. In fact, a larger gradient would imply a larger difference between the predicted peaks of the MDFs of the two sub-populations, which is not observed. However, we cannot exclude the existence of a small gradient, even between the Galactic center and Baade's window.

We computed the expected gradients for several chemical elements (Fe, $\mathrm{Mg}, \mathrm{O}, \mathrm{Si}, \mathrm{S}$, and $\mathrm{Ba}$ ) that are due to the differences between the average abundances of the two main bulge populations (MP-MR) as well as those that are due to the differences in the average abundances in the sub-populations IMP and EMP. The results are shown in Table 2. For the gradients between MP and MR as well as those between IMP and EMP, we simply show the difference between the average abundances in their MDFs.

\subsection{The evolution of Li abundance in the gas of the bulge}

We computed the evolution of the abundance of ${ }^{7} \mathrm{Li}$ in the gas out of which the two main populations (MP and MR) formed. The reason for this is that recently Bensby et al. $(2010,2011)$ measured the Li abundance in several microlensed dwarfs and subgiant stars. Their data are plotted together with our model predictions in Fig. 9. We speak of Li evolution in the gas and not in the stars because $\mathrm{Li}$ is easily destroyed inside stars and a galactic chemical evolution model aims at reproducing the upper envelope of the data in the plot $\log N(\mathrm{Li})$ vs. $[\mathrm{Fe} / \mathrm{H}]$. This procedure is commonly applied to the $\mathrm{Li}$ abundance data in the solar neighborhood stars. The nucleosynthesis prescriptions adopted here correspond to those of model $C$ of Romano et al. (1999) and references therein. In this model, the contribution from core-collapse $\mathrm{SNe}$ to ${ }^{7} \mathrm{Li}$ production is decreased by a factor of two relative to the predicted yields (Woosley \& Weaver 1995 ) and $\mathrm{Li}$ is mainly produced by massive asymptotic giant branch stars and novae. The predicted curve for the gas of the MP population reproduces very well the value of Li measured in the metal poor bulge dwarf MOA-2010-BLG-285S, which lies on the so-called Spite plateau observed in solar vicinity stars (Spite \& Spite 1982). The other values for the Li abundance are all lower than that for MOA-2010-BLG-285S, but the stars are more metal rich and the ${ }^{7} \mathrm{Li}$ in those objects has very likely been depleted. The initial value of ${ }^{7} \mathrm{Li}$ in Fig. 9 is assumed to be $\log N_{\mathrm{P}}(\mathrm{Li})=2.2$, as in Fig. 5 of the paper of Bensby et al. (2010). This initial value corresponded, until a few years ago, to what we thougth was the primordial $\mathrm{Li}$ abundance. At the present time, the situation is more complicated, since the primordial value for ${ }^{7} \mathrm{Li}$, as estimated by WMAP (Hinshaw et al. $2009)$ is $\log N_{\mathrm{P}}(\mathrm{Li}) \sim 2.6$. No convincing explanation for this discrepancy has been found so far, and the most simple interpretation of this fact is that the primordial ${ }^{7} \mathrm{Li}$ has been depleted in metal poor star. For stars with $[\mathrm{Fe} / \mathrm{H}]$ between -1.0 and $-3.0 \mathrm{dex}$, it must have been depleted by the same amount, thus creating the Spite plateau observed in the solar vicinity stars. For very low metallicities $([\mathrm{Fe} / \mathrm{H}]<-3.0 \mathrm{dex})$, the $\mathrm{Li}$ abundance could even decrease further (see Matteucci 2010, for a discussion and references therein).

\section{A chemo-dynamical model for the bulge}

Here we summarize and extend the results obtained by Pipino et al. (2010), in particular, those referring to the model labeled bulge3, in order to compare those results with the present ones. The model of Pipino et al. (2010) includes gas hydrodynamics in $1 \mathrm{D}$ and follows the evolution of the abundances of $\mathrm{O}$ and $\mathrm{Fe}$; an extensive description can be found in Pipino et al. (2008, 2010). In model bulge3, a stellar mass of $2.28 \times 10^{10} M_{\odot}$ is formed on a timescale of $\sim 0.35 \mathrm{Gyr}$ and with a Salpeter (1955) IMF, in excellent agreement with the results of Ballero et al. (2007) and Cescutti \& Matteucci (2011) as well as with the results of this paper concerning the formation of the MP population. It is worth noting that this chemo-dynamical model does not predict a bimodal population but a continuous gradient inside the bulge. The bulge forms as a result of the collapse of primordial gas. At the beginning of this kind of collapse stars form everywhere, but as the collapse proceeds, the gas accumulates towards the Galactic center. The metals tend to concentrate here due to the gas, which had been enriched by the very first stellar generations, falling towards the center. Under these conditions, an abundance gradient always forms if the collapse is not instantaneous with the more metal rich stars sitting towards the center. Due to the deep potential well of the Milky Way, no gas can escape from the bulge and the star formation stops just when the amount of gas is too low. This is in agreement with the results of this paper. The effective radius of the bulge is $\sim 1 \mathrm{kpc}$, so that by means of model bulge3, we can compute the vertical gradients between the Galactic center and $1 \mathrm{kpc}$. We find a gradient of $\frac{\Delta[\mathrm{Fe} / \mathrm{H}]}{\Delta R}=-0.36 \mathrm{dex} \mathrm{\textrm {kc } ^ { - 1 }}$ and a gradient of $\frac{\Delta[\mathrm{O} / \mathrm{Fe}]}{\Delta R}=+0.07 \mathrm{dex} \mathrm{kpc}^{-1}$. This positive gradient for the $[\mathrm{O} / \mathrm{Fe}]$ ratio is because star formation stopped earlier 
V. Grieco et al.: Chemical evolution of the Galactic bulge: different stellar populations and possible gradients

Table 2. Differences among the mean abundances in the different stellar populations, as predicted by the different models (see text); they can be interpreted as gradients.

\begin{tabular}{|c|c|c|c|c|c|c|}
\hline Model & $\Delta\langle[\mathrm{Fe} / \mathrm{H}]\rangle$ & $\Delta\langle[\mathrm{Mg} / \mathrm{H}]\rangle$ & $\Delta\langle[\mathrm{O} / \mathrm{H}]\rangle$ & $\Delta\langle[\mathrm{S} / \mathrm{H}]\rangle$ & $\Delta\langle[\mathrm{Si} / \mathrm{H}]\rangle$ & $\Delta\langle[\mathrm{Ba} / \mathrm{H}]\rangle$ \\
\hline MP-MR & $-0.521 \mathrm{dex}$ & $-0.232 \mathrm{dex}$ & $-0.214 \mathrm{dex}$ & $-0.350 \mathrm{dex}$ & $-0.325 \mathrm{dex}$ & $-0.270 \mathrm{dex}$ \\
\hline EMP-IMP & -0.145 dex & -0.142 dex & & -0.140 dex & -0.145 dex & -0.156 dex \\
\hline $\begin{array}{l}\text { bulge3 }(0-1 \mathrm{kpc}) \\
\text { bulge3 }(0-0.5 \mathrm{kpc})\end{array}$ & $\begin{array}{l}-0.36 \mathrm{dex} \mathrm{kpc}^{-1} \\
-0.26 \mathrm{dex} \mathrm{kpc}^{-1}\end{array}$ & & $\begin{array}{l}-0.29 \mathrm{dex} \mathrm{kpc}-1 \\
-0.21 \mathrm{dex} \mathrm{kpc}^{-1}\end{array}$ & & & \\
\hline
\end{tabular}

Notes. Only for model bulge3 do we show the real gradient as $\Delta\langle[\mathrm{X} / \mathrm{H}]\rangle / \Delta R$ and do we compute it for two distance ranges from the Galactic center (0-1 kpc and 0-0.5 kpc).

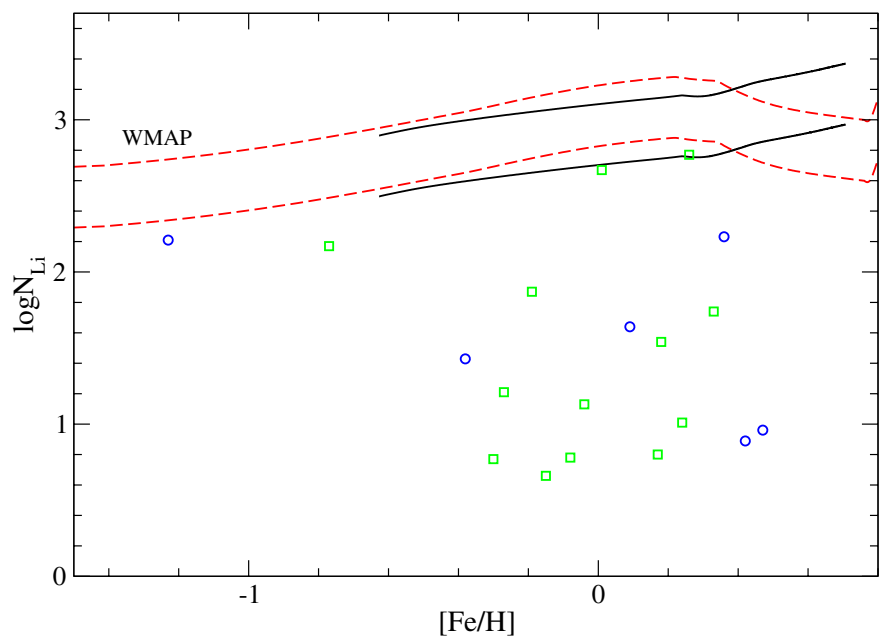

Fig. 9. Predicted evolution of Li abundance for the gas in the MP and MR populations (see details in the text) compared with $\mathrm{Li}$ abundance determinations by Bensby et al. (2011), blue circle, and by Gonzalez et al. (2009), green square. The dashed red curve refers to the MP and the continuous black curve to the MR population. The star MOA2010-BLG-285S corresponds to the point with $[\mathrm{Fe} / \mathrm{H}]=-1.23 \mathrm{dex}$ and $\log N(\mathrm{Li})=2.16$. We show also our predictions based on the assumption of the primordial Li abundance suggested by WMAP results.

in outer region, where the gas is rapidly lost because it falls towards the center. A shorter period of star formation means, in fact, higher $[\alpha / \mathrm{Fe}]$ ratios because of the less important contribution of the SNe Ia to the chemical enrichment. This predicted gradient in $[\mathrm{Fe} / \mathrm{H}]$ is certainly measurable with high-resolution spectroscopy of the bulge stars. Now, we can compare the gradient predicted by the chemo-dynamical model bulge 3 with the one obtained in this paper by assuming that the innermost region of the bulge evolved faster than the most external one. If we take the average $\langle[\mathrm{Fe} / \mathrm{H}]\rangle$ of the two sub-populations and assume that the most MP one is at $\sim 1.6 \mathrm{kpc}$ from the center, we find a gradi-

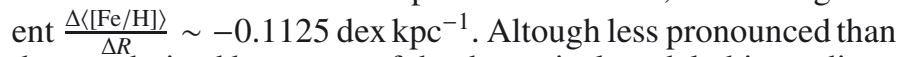
the one derived by means of the dynamical model, this gradient, if real, is still observable. The predicted gradients are reported in Table 2, where we also show the gradient predicted by the bulge 3 model from the center to $500 \mathrm{pc}$.

\section{Discussion and conclusions}

Abundance ratios are useful tools to understand the timescale for the formation of different structures. The $[\alpha / \mathrm{Fe}]$ ratios measured so far in bulge stars have indicated that a fraction of them formed on a short timescale, as indicated by the high and almost constant $[\alpha / \mathrm{Fe}]$ ratios for a large $[\mathrm{Fe} / \mathrm{H}]$ range. This means that only few stars belonging to this component formed out of gas polluted by Type Ia SNe, which occur with a time delay relative to core-collapse SNe (time-delay model). Recently, an additional population of bulge stars with average $[\mathrm{Mg} / \mathrm{Fe}] \sim 0$ and barlike kinematics has been discovered, indicating that these stars formed either on a longer timescale than the other bulge stars or out of gas already enriched and polluted by Type Ia SNe. Therefore, these indications seem to favor a complex scenario, with our bulge containing both the characteristics of a classical bulge and a pseudo-bulge. Abundance gradients have not been found in the innermost bulge region (up to $b=-4^{\circ}$ ), whereas from $b=-4^{\circ}$ to $b=-12^{\circ}$, Zoccali et al. $(2008,2009)$ and Johnson et al. (2011) found a gradient along the bulge minor axis. In this paper, we propose a scenario that can explain the existence of the MP stellar population with possible abundance gradients inside it, together with the MR bar population. Clearly, such a complex picture suggests a distinction between two different types of gradients: the gradient formed inside the MP old bulge population by dissipational collapse, and the gradient due to the differences in the average chemical abundances in the classical and pseudo-bulge populations. The gradient inside the classical bulge population should show a decrease in metallicity from the innermost to the outermost bulge regions. On the other hand, the gradient between the classical bulge (MP) and the pseudo-bulge (MR) population should be more difficult to identify since the stars of the two populations could have been mixed at any Galactic latitude.

We have run different chemical evolution models to reproduce these different populations. Our conclusions can be summarized as follows:

- Both the MDF and the abundance ratios of the MP population can be reproduced by a classical chemical evolution model for the bulge; this model suggests a formation timescale of $\sim 0.1-0.3 \mathrm{Gyr}$, an IMF flatter than in the solar vicinity, although less flat than previously suggested. In fact, the recent data can be well reproduced by a Salpeter (1955) $(x=1.35)$ IMF. We assumed that the infalling gas forming this component was pre-enriched at the level of the average metallicity of halo stars $(\langle[\mathrm{Fe} / \mathrm{H}]\rangle=-1.5 \mathrm{dex})$. If this is true, we predict that stars with $[\mathrm{Fe} / \mathrm{H}]<-1.5$ dex should not exist in the Galactic bulge.

- We also assumed that inside the classical bulge the most internal stars formed more rapidly than those more external and to simulate this effect we simply assumed that the star formation efficiency was more rapid internally $(v=$ $\left.25 \mathrm{Gyr}^{-1}\right)$ than externally $\left(v=10 \mathrm{Gyr}^{-1}\right)$, thus mimicking a dissipative gravitational collapse. Then we computed the average abundances of these two distinct sub-populations and found that the sub-population higher on the Galactic plane should be less metal rich, in agreement with what was found by Zoccali et al. (2008, 2009). We also ran a chemo-dynamical model following a dissipational collapse for the bulge formation and predicted a gradient of 
$\frac{\Delta[\mathrm{Fe} / \mathrm{H}]}{\Delta R}=-0.26 \mathrm{dex} \mathrm{kpc}^{-1}$ in the Galactocentric distance range 0-500 pc. Babusiaux et al. (2010) suggested that the change in the MDF and in the kinematics as a function of metallicity with increasing Galactic latitude is due to the MR population disappearing while moving away from the plane. If this is true, any (residual) observed gradient at $b<-6^{\circ}$ must be an intrinsic property of the MP population. Here we showed that such a gradient is expected in the monolithic dissipational assembly of the MP population.

- Then, we ran a model to explain the MR population: we assumed that these stars formed with a delay relative to those of the MP population and out of a substantially enriched gas and on a longer timescale of $\sim 3$ Gyr. We compared our results with the MDF and the $[\alpha / \mathrm{Fe}]$ ratios of the MR population of Hill et al. (2011) and found a good agreement.

- We predicted abundance gradients for $\mathrm{Fe}, \mathrm{Mg}$, and also for $\mathrm{O}, \mathrm{S}, \mathrm{Si}$, and $\mathrm{Ba}$ between the MP and MR populations. The gradients (i.e., differences in the mean abundances of the two populations) we found are quite substantial (up to $-0.7 \mathrm{dex}$ for $[\mathrm{Fe} / \mathrm{H}]$ ) and certainly observable with high-resolution spectroscopy.

- Finally, we presented predictions for the evolution of the abundance of ${ }^{7} \mathrm{Li}$ in the gas out of which the MP and MR populations formed. We found a good agreement with the abundance of Li measured in a metal poor star MOA-2010BLG-285S and suggested that to obtain this agreement one needs to decrease the ${ }^{7} \mathrm{Li}$ production by core-collapse $\mathrm{SNe}$. A negligible contribution to Li by core-collapse SNe has also been recently suggested by Prantzos (2012).

Therefore, although the existence of a bimodal population is not yet proven without doubt, in agreement with Babusiaux et al. (2010) we suggest that, from the chemical point of view, the co-existence of two main different stellar populations is possible in the Galactic bulge, one probably related to a fast gravitational collapse and the other to the existence of the bar, which appears to be a predominant feature in the bulge. Last but least, we have shown that abundance gradients inside the bulge population formed by gravitational collapse can also exist, thus suggesting the existence of even more than two stellar populations. However, only future high-resolution observations of bulge stars will help to clarify this complex scenario. If no gradients are found in the inner bulge, we will conclude that the stars formed very fast (timescale $\leq 0.1-0.3 \mathrm{Gyr}$ ), with no energy dissipation and with the same high efficiency of star formation $\left(20-25 \mathrm{Gyr}^{-1}\right)$.

Acknowledgements. We are indebted to C. Flynn and M. Nonino for providing programs for handling the comparison between predictions and data. We thank D. Romano, O. Gonzalez, R. M. Rich, and M. Schultheis for their careful reading of the manuscript and very useful suggestions and M. Zoccali for many illuminating discussions. We also thank an anonymous referee for his/her suggestions, which improved the paper.

\section{References}

Aguerri, J. A. L., Balcells, M., \& Peletier, R. F. 2001, A\&A, 367, 428 Athanassoula, E. 2005, MNRAS, 358, 1477

Babusiaux, C., Gómez, A., Hill, V., et al. 2010, A\&A, 519, A77

Bekki, K., \& Tsujimoto, T. 2011, MNRAS, 416, L60

Ballero, S. K., Matteucci, F., Origlia, L., \& Rich, R. M. 2007, A\&A, 467, 123

Bensby, T., Feltzing, S., Johnson, J. A., et al. 2010, A\&A, 512, A41

Bensby, T., Adén, D., Meléndez, J., et al. 2011, A\&A, 533, A134

Bournaud, F., Elmegreen, B. G., \& Martig, M. 2009, ApJ, 707, L1
Busso, M., Gallino, R., Lambert, D. L., Travaglio, C., \& Smith, V. V. 2001, ApJ, 557,802

Cescutti, G. 2008, A\&A, 481, 691

Cescutti, G., \& Matteucci, F. 2011, A\&A, 525, A126

Cescutti, G., François, P., Matteucci, F., Cayrel, R., \& Spite, M. 2006, A\&A, 448, 557

Cescutti, G., Matteucci, F., François, P., \& Chiappini, C. 2007, A\&A, 462, 943

Cescutti, G., Matteucci, F., McWilliam, A., \& Chiappini, C. 2009, A\&A, 505, 605

Chiappini, C., Matteucci, F., \& Gratton, R. 1997, ApJ, 477, 765

Chiappini, C., Matteucci, F., \& Romano, D. 2001, ApJ, 554, 1044

Clarkson, W., Sahu, K., Anderson, J., et al. 2008, ApJ, 684, 1110

Clarkson, W. I., Sahu, K. C., Anderson, J., et al. 2011, ApJ, 735, 37

Combes, F., Debbasch, F., Friedli, D., \& Pfenniger, D. 1990, A\&A, 233, 82

Ferreras, I., Wyse, R.F.G., \& Silk, J. 2003, MNRAS, 345, 1381

François, P., Matteucci, F., Cayrel, R., et al. 2004, A\&A, 421, 613

Gonzalez, O. A., Zoccali, M., Monaco, L., et al. 2009, A\&A, 508, 289

Gonzalez, O. A., Rejkuba, M., Zoccali, M., et al. 2011, A\&A, 530, A54

Johnson, C. I., Rich, R. M., Fulbright, J. P., Valenti, E., \& McWilliam, A. 2011, ApJ, 732, 108

Johnson, C. I., Rich, R. M., Kobayashi, C., \& Fulbright, J. P. 2012, ApJ, 749, 175

Hill, V., Lecureur, A., Gómez, A., et al. 2011, A\&A, 534, A80

Hinshaw, G., Weiland, J. L., Hill, R. S., et al. 2009, ApJS, 180, 225

Kormendy, J., \& Kennicutt, R. C., Jr. 2004, ARA\&A, 42, 603

Kuijken, K., \& Rich, R. M. 2002, AJ, 124, 2054

Kunder, A. M., de Propris, R., Rich, M., et al. 2011, BAAS, 43, AAS Meeting, $217,241.12$

Lanfranchi, G. A., Matteucci, F., \& Cescutti, G. 2006, MNRAS, 365, 477

Larson, R. B. 1976, MNRAS, 176, 31

Maeder, A. 1992, A\&A, 264, 105

Matteucci, F. 2010, in Light elements in the Universe, eds. C. Charbonnel et al., Proc. IAU Symp., 268, 453

Matteucci, F., \& Brocato, E. 1990, ApJ, 365, 539

Matteucci, F., \& Romano, D. 1999, Ap\&SS, 265, 311

Matteucci, F., Romano D., \& Molaro P. 1999, A\&A, 341, 458

McWilliam, A., \& Zoccali, M. 2010, ApJ, 724, 1491

McWilliam, A., Matteucci, F., Ballero, S., et al. 2008, AJ, 136, 367

Meynet, G., \& Maeder, A. 2002, A\&A, 390, 561

Minniti, D., Olszewski, E. W., Liebert, J., et al. 1995, MNRAS, 277, 1293

Nataf, D. M., Udalski, A., Gould, A., Fouqué, P., \& Stanek, K. Z. 2010, ApJ, 721, L28

Ness, M., \& Freeman, K. 2012, EPJ Conf., 19, 06003 (EDP Sciences)

Noguchi, M. 1999, ApJ, 514, 77

Norman, C. A., Sellwood, J. A., \& Hasan, H. 1996, ApJ, 462, 114

Pipino, A., \& Matteucci, F. 2004, MNRAS, 347, 968

Pipino, A., D'Ercole, A., \& Matteucci, F. 2008, A\&A, 484, 679

Pipino, A., D'Ercole, A., Chiappini, C., \& Matteucci, F. 2010, MNRAS, 407, 1347

Prantzos, N. 2012, A\&A, 542, A67

Rahimi, A., Kawata, D., Brook, C. B., \& Gibson, B. K. 2010, MNRAS, 401, 1826

Ramírez, S. V., Stephens, A. W., Frogel, J. A., \& DePoy, D. L. 2000, AJ, 120 833

Rich, R. M., Reitzel, D. B., Howard, C. D., \& Zhao, H. S. 2007a, ApJ, 658, L29

Rich, R. M., Origlia, L., \& Valenti, E. 2007b, ApJ, 665, L119

Rich, R. M., Origlia, L., \& Valenti, E. 2012, ApJ, 746, 59

Robin, A. C., Marshall, D. J., Schultheis, M., \& Reylé, C. 2012, A\&A, 538, A106

Romano, D., Matteucci, F., Molaro, P., \& Bonifacio, P. 1999, A\&A, 352, 117

Salpeter, E. E. 1955, ApJ, 121, 161

Saito, R. K., Zoccali, M., McWilliam, A., et al. 2011, AJ, 142, 76

Samland, M., \& Gerhard, O. E. 2003, A\&A, 399, 961

Scalo, J. M. 1986, FCPh, 11, 1

Shen, J., Rich, R. M., Kormendy, J., et al. 2010, ApJ, 720, L72

Spite, F., \& Spite, M. 1982, A\&A, 115, 357

Tsujimoto, T., \& Bekki, K. 2012, ApJ, 747, 125

Uttenthaler, S., Schultheis, M., Nataf, D. M., et al. 2012, A\&A, 546, A57

van den Hoek, L. B., \& Groenewegen, M. A. T. 1997, A\&AS, 123, 305

Woosley, S. E., \& Weaver, T. A. 1995, ApJ, 101, 181

Wyse, R. F. G., \& Gilmore, G. 1992, AJ, 104, 144

Zoccali, M., Renzini, A., Ortolani, S., et al. 2003, A\&A, 399, 931

Zoccali, M., Hill, V., Lecureur, A., et al. 2008, A\&A, 486, 177

Zoccali, M., Hill, V., Barbuy, B., et al. 2009, The Messenger, 136, 48 